\title{
Simplification of Calculations in Routine Density and Volumetric Determinations
}

\author{
Charles T. Collett
}

\begin{abstract}
In density and volumetric determinations, certain methods of weighing have encouraged the use of approximations. These can be avoided by minor changes in procedure that simplify calculations. The use of counterpoises is shown to eliminate some buoyancy corrections.
\end{abstract}

\section{Introduction}

In the course of performing numerous density and volumetric determinations several methods were compared with regard to the calculations associated with them.

Some methods involved equations that were found adequate and correct but too complicated to be desirable for routine work. For example, Gould and Stott ${ }^{1}$ represent the density of a liquid in a glass picnometer by an equation of 26 terms and some 37 brackets, parentheses, shillings, etc.

Other systems were observed to lead the worker into dilemmas, from which he escaped by the use of approximations. These did not reduce the accuracy of his computed values, but were time-consuming and difficult to record in concise form. Often the steps of the calculations were worked out on scratch paper and then discarded, only the terminal values of the series being permanently recorded.

The methods and equations finally selected for everyday use were derived from inspection of the component forces acting on the arms of a balance in equilibrium, i. e., forces resulting from the masses pushing downward and the buoyant effects pushing upward. Because these equations are based on considerations that are elementary to the problem, it would seem highly probable that they have been used before, and perhaps published. A search of the literature has not revealed them, at least not in the forms shown here. Certainly, they have been neglected in modern practice, and their simplicity and usefulness suggest that they should be presented for consideration.

\section{Hydrostatic Weighing}

Consider that it is desired to find the mass, $M_{s}$, of a glass object of irregular shape by the conventional substitution weighing. Assume that at all times a constant load, $c$, remains on the right pan of the laboratory balance, a condition that will be unchanged during all weighings subsequently discussed, and the designation as right or left being an arbitrary identification without other significance.

F. A. Gould and Verney Stott, Glazebrook's dictionary of applied physies, III, 127 (Peter Smith, New York, N. Y., 1950).
Equilibrium is established by placing on the left pan brass weights $e$ and $f$, whose masses are $M_{e}$ and $M_{f}$ and which are subject to air-buoyancy effects, $B_{e}$ and $B_{f} . \quad M_{c}$ is the mass of $c$, and $B_{c}$ is the air buoyancy on $c$. Then

$$
M_{e}-B_{e}+M_{f}-B_{f}=M_{c}-B_{c} .
$$

If the glass specimen, $s$, is then placed on the left pan, and equilibrium restored by removing weight $f$,

$$
M_{e}-B_{e}+M_{s}-B_{s}=M_{c}-B_{c},
$$

where $M_{s}$ represents the mass of the specimen and $B_{s}$ the air buoyancy acting upon it. The second weighing must be accomplished within a reasonably short time, so that the air density has not changed. Subtracting eq (2) from eq (1), and rearranging,

$$
\begin{aligned}
M_{s} & =M_{f}-B_{f}+B_{s} \\
& =M_{f}-\frac{M_{f}}{\rho_{f}} \rho_{a}+V_{s} \rho_{a} \\
& =M_{f}\left(1-\frac{\rho_{a}}{\rho_{f}}\right)+V_{s} \rho_{a},
\end{aligned}
$$

where $\rho_{a}$ is the density of the air, $\rho_{f}$ is the density of the weights, and $V_{s}$ is the volume of the specimen. $\rho_{a}$ can be obtained by well-known methods, ${ }^{2}$ and $\rho_{f}$ is usually known. However, $V_{s}$ is unknown due to the shape of the specimen and is customarily found by means of hydrostatic weighing.

A suspension is fitted below the left pan to support a basket immersed in a liquid, usually distilled water, and equilibrium is secured by the addition of weights $g$ and $h$ on the pan. Let $\dot{M}_{k}$ equal the mass of the suspension system and $B_{k}$ and $B^{\prime}{ }_{k}$ the respective air and water buoyancy effects.

$$
M_{g}-B_{o}+M_{h}-B_{h}+M_{k}-B_{k}-B^{\prime}{ }_{k}=M_{c}-B_{c} .
$$

The next operation must be performed within a short period of time, that is, before the density of the air or the water has changed appreciably. The speci-

${ }^{2}$ N. Bauer, Technique of organic chemistry, vol. I, part 1, Physical Methods, p. 273 (Interscience Publishers, Inc., New York, N. Y., 1949). N. S. Osborne, E. C. McKelvey, and H. W. Bearce, Density and thermal expansion of ethy alcohol and of its mixtures with water, Bul. BS 9, 327 (1913) S197 (see p. 378). 
men is immersed within the basket and is subject to buoyancy effect $B^{\prime}{ }_{s}$. Weight $h$ is removed to restore equilibrium. Then

$$
M_{g}-B_{g}+M_{k}-B_{k}-B^{\prime}{ }_{k}+M_{s}-B^{\prime}{ }_{s}=M_{c}-B_{c} .
$$

Subtracting eq (5) from eq (4)

$$
\begin{aligned}
B_{s}^{\prime} & =M_{s}-M_{h}+B_{h} \\
& =M_{s}-M_{h}+\frac{M_{h}}{\rho_{h}} \rho_{a}^{\prime} \\
& =M_{s}-M_{h}\left(1-\frac{\rho_{a}^{\prime}}{\rho_{h}}\right) .
\end{aligned}
$$

Again an unknown, $M_{s}$, appears on the right side of the equation, making it impossible to use directly the relationship

$$
\begin{aligned}
V_{s}= & \frac{B_{s}^{\prime}}{\rho_{w}} \\
& =\frac{M_{s}-M_{h}\left(1-\frac{\rho_{a}^{\prime}}{\rho_{h}}\right)}{\rho_{w}},
\end{aligned}
$$

where $\rho_{v}$ is the density of the water. ${ }^{3}$

The unknown quantities may be eliminated from the right-hand sides of eq (3) and (7) by substitution. The resulting equations can be solved directly but contain more terms, thus:

$$
\begin{gathered}
M_{s}=\frac{M_{f}\left(1-\frac{\rho_{a}}{\rho_{f}}\right)-M_{h}\left(\frac{\rho_{a}}{\rho_{w}}\right)\left(1-\frac{\rho_{a}^{\prime}}{\rho_{f}}\right)}{1-\frac{\rho_{a}}{\rho_{w}}} . \\
V_{s}=\frac{M_{f}\left(1-\frac{\rho_{a}}{\rho_{f}}\right)-M_{h}\left(1-\frac{\rho_{a}^{\prime}}{\rho_{f}}\right)}{\rho_{w}-\rho_{a}} .
\end{gathered}
$$

However, if eq (9) is solved first, the value of $V_{s}$ may be used in eq (3), which is much shorter.

Equations (3) and (7) may be solved by the use of approximations, a method that has been found in considerable use. ${ }^{4}$ In a typical case it was found necessary to remove weights equal to $11.6954 \mathrm{~g}$ after the glass sample was placed on the pan. Air density was determined to be $0.001170 \mathrm{~g} / \mathrm{ml}$, and the weights were known to have a density of 8.4 $\mathrm{g} / \mathrm{cm}^{3}$. From eq (3):

$$
\begin{aligned}
M_{s} & =11.6954\left(1-\frac{0.001170}{8.4}\right)+V_{s}(0.001170) \\
& =11.6938+V_{s}(0.001170), \text { in grams. }
\end{aligned}
$$

For the hydrostatic weighing, equilibrium was maintained by removing $6.4711 \mathrm{~g}$ after the sample was placed in the immersed basket. Densities of

${ }^{3}$ A similar equation appears in Bul. BS 9, 327 (1913) S197, see p. 384, where the density of a liquid is found by immersing a sinker of known mass and volume.

${ }_{4}$ NBS Circular 487, p. 2 (1950). the water and air were $0.996953 \mathrm{~g} / \mathrm{ml}$ and 0.001176 $\mathrm{g} / \mathrm{ml}$. From eq (7)

$$
\begin{aligned}
V_{s} & =\frac{M_{s}-6.4711\left(1-\frac{0.001176}{8.4}\right)}{0.996953} \\
& =\frac{M_{s}-6.4702}{0.996953} \mathrm{ml} .
\end{aligned}
$$

The first approximation is secured by using the apparent weight of the sample in place of $M_{s}$ in eq (11), giving

$$
\operatorname{approx}_{\cdot 1} V_{s}=\frac{11.6954-6.4702}{0.996953}=5.2412 \mathrm{~cm}^{3} \text {. }
$$

This approximate value for $V_{s}$ is used in eq (10), and we have

$\operatorname{approx}_{.1} M_{s}=11.6938+5.2412(0.001170)=11.6999 \mathrm{~g}$.

The second approximations are

$$
\operatorname{approx} .2_{s} V_{s}=\frac{11.6999-6.4702}{0.996953}=5.2457 \mathrm{~cm}^{3}
$$

approx.2 $M_{s}=11.6938+5.2457(0.001170)=11.6999 \mathrm{~g}$.

These last approximate values are accepted for the real values, as obviously no further change will occur.

A minor alteration in this procedure will simplify the equations and entirely eliminate the use of approximations. The suspended, immersed basket is retained, but the specimen rests on the left pan during the first weighing, and equilibrium is produced by the presence of weight $g$. Equation (4) then becomes

$$
M_{g}-B_{g}+M_{s}-B_{s}+M_{k}-B_{k}-B_{k}^{\prime}=M_{c}-B_{c} .
$$

For the second weighing the specimen is transferred to the basket to be immersed as before, but to produce equilibrium it is necessary to add weight $h$ on the pan. ${ }^{5}$ Equation (5) then is changed to:

$$
\begin{aligned}
& M_{g}-B_{g}+M_{h}-B_{h}+M_{k}-B_{k}- \\
& B_{k}^{\prime}+M_{s}-B_{s}^{\prime}=M_{c}-B_{c} .
\end{aligned}
$$

Subtracting eq (17) from eq (16)

$$
\begin{gathered}
B_{s}^{\prime}-B_{s}=M_{h}-B_{h} \\
V_{s}\left(\rho_{w}-\rho_{a}\right)=M_{h}-\frac{M_{h}}{\rho_{h}} \rho_{a} \\
V_{s}=\frac{M_{h}\left(1-\frac{\rho_{a}}{\rho_{h}}\right)}{\rho_{w}-\rho_{a}} .
\end{gathered}
$$

5 The transfer of sample from pan to basket is described by W. Watson (A textbook of physies, p. 175, Longmans, Green \& Co., London, 1902), but the calculations are not mentioned. 
Because the densities of weight $h$, air, and water can be secured easily, it is apparent that eq (18) contains no unknowns on the right-hand side, and the exact value of $V_{s}$ may be calculated at once. After its volume is thus determined, the object is weighed in air in the conventional manner, after which eq (3) can be solved.

The same glass piece previously considered was also weighed by this latter method. After the sample was moved from the pan to the immersed basket, it was necessary to place $5.2243 \mathrm{~g}$ on the pan. The densities of the air and water were respectively 0.001170 and $0.996953 \mathrm{~g} / \mathrm{ml}$, and of the weights, $8.4 \mathrm{~g} / \mathrm{cm}^{3}$

From eq (18)

$$
V_{s}=\frac{5.2243\left(1-\frac{0.001170}{8.4}\right)}{0.996953-0.001170}=5.2457 \mathrm{~cm}^{3} .
$$

The sample was then weighed in air in the conventional substitution method, $11.6954 \mathrm{~g}$ being removed after the specimen was placed on the pan. Air density was $0.001170 \mathrm{~g} / \mathrm{ml}$.

From eq (3):

$$
M_{s}=11.6954\left(1-\frac{0.001170}{8.4}\right)+5.2457(0.001170)
$$

$=11.6999 \mathrm{~g}$.

\section{Weighing a Picnometer by the Counter- poise Method}

Weighing a picnometer is a special case of determining the mass of a glass object having irregular shape and unknown volume, and is therefore subject to the same general principles. If an attempt is made to use conventional substitution weighing, the same dilemma will of course arise, that is, neither the mass nor the volume is known, and neither can be determined without knowledge of the other. The volume here referred to is the volume of material (glass) that will displace air during the weighing, and does not refer to the capacity of the container, or so-called "internal volume."

Furthermore, the interior of the picnometer for liquids is of necessity closed to the outside except for limited entrances, usually capillary tubes of small diameter. For this reason, filling, emptying, cleaning, and drying are not quickly accomplished, and the observer will wish to hold the number of such operations to a minimum. For example, a series of trials may be performed while the same liquid remains in the instrument, only minor adjustments of level being made between succeeding weighings.

Thus the weighing of the empty picnometer and the weighing of the filled picnometer are often separated by considerable periods of time, and the air density, and hence the air buoyancy effects, will vary.

However, the user of the picnometer is not primarily interested in the mass of the instrument; this value is never required except as a tare, and its consistent treatment as such throughout all operations will permit the use of simple calculations with no approximations. By the use of a counterpoise the picnometer's mass and its buoyancy in air may be entirely eliminated from all calculations, and in fact need never be known at any time. ${ }^{6}$

The counterpoise is a second picnometer similar in size and shape to the first, made of glass with the same density, and the two instruments must be approximately equal in mass. A small protuberance is provided on each, such as the handle on the cap, and by grinding and polishing it is not difficult to adjust the masses within a milligram. However, the two instruments may be allowed to differ by as much as $0.1 \mathrm{~g}$ because the difference in buoyant effects when they are suspended from opposite balance pans with equilibrium maintained by weights is $\left(\Delta / \rho_{g}-\Delta /\right.$ $\left.\rho_{f}\right) \rho_{a}$, where $\Delta$ is the difference between the masses of the two picnometers, and $\rho_{g}, \rho_{f}$, and $\rho_{s}$ are the densities of the glass, weights, and air. If $\Delta$ is $0.1 \mathrm{~g}$ and the respective densities are about 2.5, 8.4, and $0.0012 \mathrm{~g} / \mathrm{ml}$, the difference in buoyant effect will be negligible.

In conventional laboratory practice the mass, volume of glass, and internal volume of a picnometer are not usually determined each time the instrument is used, but only at the time it is calibrated. With occasional checking, these values are considered true as long as there is no reason to suspect a change. In the method to be considered, only the internal volume at various temperatures is determined.

The counterpoise is placed upon the right pan with a constant load composed of weights $e$ and $f$, and the active picnometer is placed on the left pan with weights $E$ and $F$ sufficient to secure a state of balance, from which the equation may be written

$$
\begin{aligned}
& M_{P}-B_{P}+M_{E}-B_{E}+M_{F}-B_{F}= \\
& M_{p}-B_{p}+M_{e}-B_{e}+M_{f}-B_{f},
\end{aligned}
$$

where $M$ is the mass, and $B$ is the air buoyancy effect of the object indicated by the subscript letter; $P$ and $p$ being the active picnometer and the counterpoise picnometer.

The picnometer is next filled with a sample, $S$, which will be distilled water during the calibration and later the particular liquid being tested for density. Balance is restored by removing weight $F$. Then

$$
\begin{aligned}
M_{P}-B_{P}^{\prime \prime}+M_{S}- & B_{S}^{\prime \prime}+M_{E}-B_{E}^{\prime \prime}= \\
& M_{p}-B_{p}^{\prime \prime}+M_{e}-B_{e}^{\prime \prime}+M_{f}-B_{f}^{\prime \prime} .
\end{aligned}
$$

It has been observed that the two weighings may be performed at different times, thus a change in air density is to be expected. The buoyant effects in eq (22) will not be equal to those in eq (21) except by coincidence and are thus designated as $B^{\prime \prime}$. ${ }^{6} \mathrm{~N}$. Bauer, Technique of organic chemistry, I, part 1, Physical methods,
p. 272 (Interscience Publishers, Inc., New York, N. Y., 1949). 
Subtracting eq (22) from eq (21), we have

$$
\begin{aligned}
& M_{F}-B_{F}-B_{P}-B_{E}+B_{P}^{\prime \prime}+B_{E}^{\prime \prime}-M_{S}+B_{S}^{\prime \prime}= \\
& B_{p}^{\prime \prime}+B_{e}^{\prime \prime}+B_{f}^{\prime \prime}-B_{p}-B_{e}-B_{f} .
\end{aligned}
$$

Assume that the small difference in mass between $P$ and $p$ in eq (21) and (22) has been compensated by a corresponding difference in $E$ and $e$, so that

$$
M_{P}+M_{E}=M_{p}+M_{e} .
$$

It has been shown that the buoyancy effects may be considered equal, that is,

Also

$$
B_{P}+B_{E}=B_{p}+B_{e}
$$

$$
B_{P}^{\prime \prime}+B_{E}^{\prime \prime}=B_{p}^{\prime \prime}+B_{e}^{\prime \prime} .
$$

It follows that $M_{F}$ must be equal to $M_{f}$, and as the weights have the same density, $B_{F}$ and $B_{f}$ are equal.

We then have

$$
M_{S}-B_{S}^{\prime \prime}=M_{F}-B_{f}^{\prime \prime},
$$

which is essentially the same as eq (3).

$$
M_{S}-\frac{M_{S}}{\rho_{S}} \rho_{a}^{\prime \prime}=M_{F}-\frac{M_{F}}{\rho_{F}} \rho_{a}^{\prime \prime},
$$

where $\rho_{a}^{\prime \prime}$ is the air density corresponding to eq (22).

$$
M_{S}=M_{F} \frac{1-\frac{\rho_{a}^{\prime \prime}}{\rho_{F}}}{1-\frac{\rho_{a}^{\prime \prime}}{\rho_{S}}} .
$$

As the sample in the picnometer during calibration is water at a known temperature, the density $\rho_{S}$ is read from standard tables, and the volume then is $M_{S}$ divided by that density. This process is repeated at different temperatures until a calibration curve has been developed for the picnometer over the desired range.

When a liquid of unknown density is to be tested the weighing procedure is exactly the same. However, eq (27), as shown in eq (3), can be rewritten to obtain

$$
M_{S}=M_{F}\left(1-\frac{\rho_{a}^{\prime \prime}}{\rho_{F}}\right)+V_{S} \rho_{a}^{\prime \prime} .
$$

The picnometer having been previously calibrated, $V_{S}$ is readily determined and

$$
\rho_{S}=\frac{M_{S}}{V_{S}}=\frac{M_{F}\left(1-\frac{\rho_{a}^{\prime \prime}}{\rho_{F}}\right)+V_{S} \rho_{a}^{\prime \prime}}{V_{S}} .
$$

For example, when a picnometer was calibrated according to the method described, a weight of $120.9878 \mathrm{~g}$ was on the left pan with the empty instrument, and $21.1467 \mathrm{~g}$ when it was filled with distilled water at a temperature of $24.288^{\circ} \mathrm{C}$. The densities of the weights, air, and water were determined to be $8.4 ; 0.001170$, and $0.997257 \mathrm{~g} / \mathrm{ml}$, respectively. From eq (29) the mass of the water was

$$
M_{S}=99.8411 \frac{1-\frac{0.001170}{8.4}}{1-\frac{0.001170}{0.997257}}=99.9445 \mathrm{~g} .
$$

The volume of the instrument was

$$
V_{S}=\frac{99.9445}{0.997257}=100.2194 \mathrm{ml} .
$$

This was used as one point on a calibration curve.

When the instrument was used to test a sample of heptane a weight of $116.5413 \mathrm{~g}$ was required on the left pan with the empty picnometer. It was then filled with the sample at a temperature of $24.992^{\circ} \mathrm{C}$, and balance was restored by reducing the weight on the pan to $48.5356 \mathrm{~g}$. The densities of the weights and the air were 8.4 and $0.001173 \mathrm{~g} / \mathrm{ml}$, respectively. From the previously drawn calibration curve the volume of the instrument at that temperature was known to be $100.2201 \mathrm{ml}$.

Using eq (31), the density of the heptane was

$$
\begin{aligned}
\rho_{S} & =\frac{68.0057\left(1-\frac{0.001173}{8.4}\right)+(100.2201)(0.001173)}{100.2201} \\
& =0.67964 \mathrm{~g} / \mathrm{ml} .
\end{aligned}
$$

Washington, October 19, 1953. 